NISSUNA UMANA INVESTIGAZIONE SI PUO DIMANDARE VERA SCIENZIA S'ESSA NON PASSA PER LE MATEMATICHE DIMOSTRAZIONI LEONARDO DA VINCI

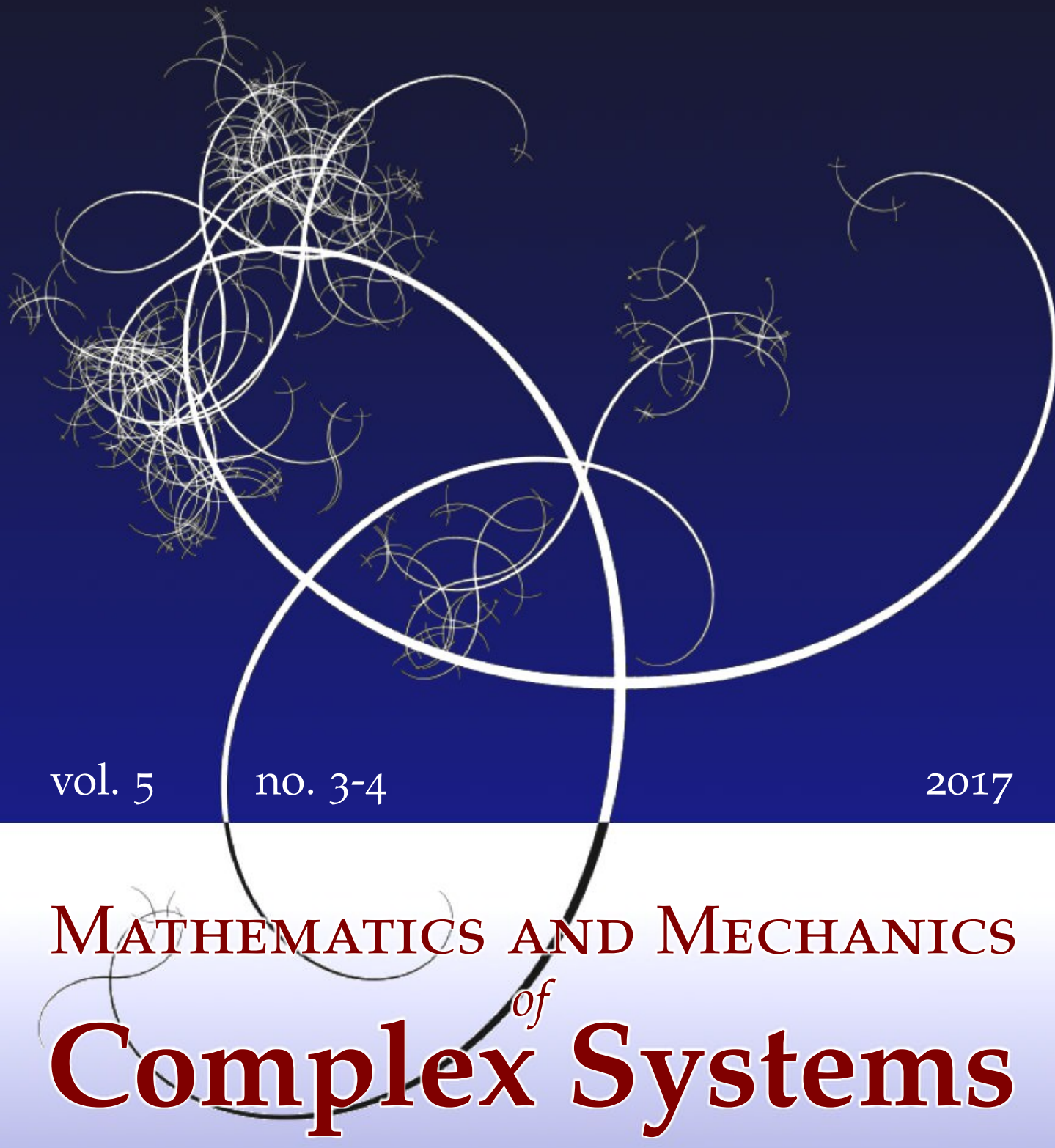

RUBEN INTERIAN, REINALDO RODRÍGUEZ-RAMOS, FERnANDo VALDÉS-RAVELO, ARIEL RAMÍREZ-TORRES, Celso C. Ribeiro and Aura Conci TUMOR GROWTH MODELLING BY CELLULAR AUTOMATA 


\title{
TUMOR GROWTH MODELLING BY CELLULAR AUTOMATA
}

\author{
RUBEn INTERIAN, REINALDO RODRÍGUEZ-RAMOS, \\ FERnando VAldés-RAVElo, ARIEl Ramírez-Torres, \\ Celso C. Ribeiro AND Aura CONCI
}

\begin{abstract}
Tumor growth is a complex process that requires mathematical modeling approaches for studying real-life cancer behavior. The use of cellular automata (CA) to represent tumor growth in its avascular stage is explained in this work, and a stochastic CA describing tumor growth is obtained, based on a differential equations system in the range of continuum mechanics. The novelty of this research is the deduction of the neighborhood structure and rules for a probabilistic CA from these differential equations that describe the evolution of the tumor growth. In addition, the influence of the stresses on tumor growth is captured by the CA.
\end{abstract}

\section{Introduction}

Cell-based and cell-centered approaches for the study of biological soft tissues have been widely used [Hoehme and Drasdo 2010; Merks and Glazier 2005]. In particular, cellular automata (CA) are one of the most successful models [Rejniak and Anderson 2011; Boondirek et al. 2010] and have been used in a large number of studies [Kansal et al. 2000b; Dormann and Deutsch 2002; Deutsch and Dormann 2005].

Cellular automata models have been widely used to simulate avascular tumor growth [Dormann and Deutsch 2002; Kansal et al. 2000a], tumor cell invasion [Anderson et al. 2009], and tumor interactions with various environmental factors [Rejniak and McCawley 2010]. A review of the main methodologies for CA models describing tumor growth is provided in [Boondirek et al. 2010], emphasizing that most researchers have attempted to consider a microscopic scale to describe the macroscopic characteristics of tumor morphology. In [Kansal et al. 2000b], a three-dimensional cellular automaton model of brain tumor growth is developed, simulating the Gompertzian model very precisely. A quantitative analysis of the growth of a subpopulation within a previously homogeneous tumor is presented in

\section{Communicated by Francesco dell'Isola.}

MSC2010: 37B15, 68Q80, 74A10.

Keywords: tumor growth, stresses, stochastic rules, cellular automata. 
[Kansal et al. 2000a], applying a cellular automaton with a Delaunay triangulation lattice. In [Dormann and Deutsch 2002], avascular tumor growth is simulated using a hybrid lattice-gas cellular automaton, exhibiting self-organized formation of a layered tumor structure. A multiscale investigation focused on tumor cell invasion is given in [Anderson et al. 2009], using an evolutionary hybrid cellular automata model (EHCA). A comparison of relative strengths and weaknesses of various cellbased models, including CA, is presented in [Rejniak and McCawley 2010].

In this work, cellular automata are used for studying the process of tumor growth, specially in its avascular stage. The novelty of this research is the deduction of the neighborhood structure and the rules for a stochastic CA from continuous deterministic differential equation (DE) models. In addition, both the application of CA to study the evolution of tumor growth in time and the influence of the stresses in the growing of the tumor are considered in this work. The validation of the theoretical biomechanical model using CA is presented as well.

Having a CA model created from a system of differential equations allows one to describe more realistically the real-life situation of the tumor stage. The goals of the present work are summarized as follows:

(1) To visualize the tumor growth process represented by a system of differential equations.

(2) To obtain the evolution of the variables over time, mainly the tumor radius, in a new manner, more realistic and closer to the reality, where the tumor is not perfectly regular or circular. To reach this goal, a continuous deterministic DE model is transformed into a probabilistic CA.

(3) To create an alternative for studying the process of tumor growth in an interactive mode.

\section{Cellular automata}

Cellular automata have been seen mainly as discrete abstract computational systems. The Stanford Encyclopedia of Philosophy [Berto and Tagliabue 2012] defines cellular automata as follows:

Firstly, CA are (typically) spatially and temporally discrete: they are composed of a finite or denumerable set of homogeneous, simple units, the atoms or cells. At each time unit, the cells instantiate one of a finite set of states. They evolve in parallel at discrete time steps, following state update functions or dynamical transition rules: the update of a cell state obtains by taking into account the states of cells in its local neighborhood (there are, therefore, no actions at a distance). Secondly, CA are abstract, as they can be specified in purely mathematical terms and implemented 
in physical structures. Thirdly, CA are computational systems: they can compute functions and solve algorithmic problems. Despite functioning in a different way from traditional, Turing machine-like devices, CA with suitable rules can emulate a universal Turing machine, and therefore compute, given Turing's Thesis, anything computable.

Rigorously, the cellular automata is a quadruple $(C, n, S, f)$ [Deutsch and Dormann 2005], where:

- $C$ is a set of cells, not required to be finite.

- $n: C \times C \rightarrow\{0,1\}$ is a neighborhood function that can been seen as a relationship (usually reflexive and symmetric) between the cells. This function shows which pairs of cells are neighbors, that is, the geometry of the cell organization. Furthermore, $n$ must satisfy the neighborhood size independence condition: $\left|N\left(c_{0}\right)\right|=\left|\left\{c \in C: n\left(c_{0}, c\right)=1\right\}\right|=N$ is a constant for every $c_{0} \in C$; i.e., the size of the neighborhood is the same for all cells.

- $S$ is a set of states. As discussed below, each cell will have an associated state, in each moment.

- $f: S^{|N|} \rightarrow S$ is a transition function. The transition function is a core of the CA dynamics and is commonly expressed with rules that define the state of the cell in the next time moment from the state of the cell neighbors.

The set of cells $C$ with the neighborhood function $n$ defines the structure of the cell space.

The simplest CA model can have binary cells (two states, "tumoral" or "normal" [Hu and Ruan 2003]). Commonly, the states are represented by a set of integer values $\{0,1,2, \ldots\}$, each of these values having an appropriate physical or biological interpretation.

\section{Linear elasticity tumor model}

This work focuses on the mathematical model developed in [Ramírez-Torres et al. 2016], where the authors using linear elasticity generalize the Ngwa-Agyngi model [2012], which describes the evolution of growth-induced stresses in a spherical and isotropic growing tumor surrounded by an external medium.

The generalization of the model considering real cases of stresses is discussed in [Ramírez-Torres et al. 2016], where the dependence of tumor growth on the stresses is analytically derived. This particular model explores the avascular stage of a solid tumor.

This section briefly describes the model. We note that, in this article, we do not focus on its derivation but, instead, on obtaining from the model a CA that represents the tumor growth, as described in the next section. 


\begin{tabular}{|cl|}
\hline Variable & Definition \\
\hline$t$ & Time \\
$r$ & Radial coordinate \\
$R(t)$ & Radius of the tumor at time $t$ \\
$R_{0}$ & Radius of the tumor at $t=0$ \\
$c(r, t)$ & Nutrient concentration inside the tumor \\
$c_{b}$ & Nutrient concentration at boundary \\
$\boldsymbol{u}(r, t)$ & Tumor cell displacement \\
$\boldsymbol{v}(r, t)$ & Tumor cell displacement velocity \\
$\boldsymbol{\sigma}$ & Cauchy stress tensor of the tumor \\
$\sigma_{r}$ & Cauchy stress tensor component in radial direction \\
$\sigma_{\theta}$ & Cauchy stress tensor component in transversal direction \\
$\boldsymbol{\sigma}^{e}$ & Cauchy stress tensor of the external medium \\
$E$ & Young's modulus \\
$\nu$ & Poisson's ratio \\
$\boldsymbol{e}$ & Strain tensor of the tumor \\
$\boldsymbol{e}^{e}$ & Strain tensor of the external medium \\
$\rho$ & Tumor cell density \\
$\gamma_{r}$ & Tumor growth anisotropy parameter (radial direction) \\
$\gamma_{\theta}$ & Tumor growth anisotropy parameter (transversal direction) \\
$\eta_{1}$ & Dependence of cell proliferation on stresses \\
$\eta_{2}$ & Dependence of cell death on stresses \\
&
\end{tabular}

Table 1. Model variable definitions.

The variables and constants of the model are shown in Table 1.

The mathematical model obtained by [Ramírez-Torres et al. 2016] is a generalization of [Ngwa and Agyingi 2012] as a result of the followings assumptions:

(i) Tumor cells form a homogeneous population that is considered a continuum.

(ii) There is adhesion between tumor cells at the boundary, which maintains the tumor's solid shape and is in equilibrium with the expansive forces exerted by the internal cell proliferation.

(iii) The tumor has a spherical shape, and its symmetry is maintained at all times.

(iv) The tumor is in a state of diffusion equilibrium.

(v) The nutrient consumption rate is proportional to the nutrient concentration and to the tumor cell density. Without stresses, the cellular proliferation rate is proportional to the nutrient concentration and to the tumor cell density, while cell death is proportional to the cellular density.

(vi) The tumor material is assumed to be incompressible and responds to stress in a purely elastic and isotropic form.

(vii) There is a constant nutrient concentration in the tumor boundary. 
(viii) There is an external medium, which is supposed to be elastic, isotropic, and incompressible.

The tumor is modeled as a solid in the three-dimensional space, and the forces on it are considered acting per volume unit. As a result of the spherical symmetry hypothesis, the problem is treated in one dimension with respect to the radial coordinate $r$.

3.1. Kinematics and equilibrium equations. Because of the radial symmetry (hypothesis (iii)), the surface of the tumor is given by $S=r-R(t)$ and the velocity field has the form $\boldsymbol{v}=\left(v_{r}, 0,0\right)$, leading to

$$
\frac{d R}{d t}=v_{r}(R, t) \text {. }
$$

This equation represents the growth rate of the tumor. Removing the inertial factors and considering hypothesis (i), the equilibrium equation is

$$
\nabla \cdot \boldsymbol{\sigma}+\boldsymbol{F}=0
$$

where $\boldsymbol{F}$ is the vector of body forces, which is considered null.

3.2. Constitutive equation. The constitutive relation, which associates the stress $\sigma_{i j}$ with the material strain $e_{i j}$, represents a material with a linear elastic response subject to an anisotropic growth:

$$
e_{i j}=g\left(\delta_{1 i} \gamma_{r}+\left(\delta_{2 i}+\delta_{3 i}\right) \gamma_{\theta}\right) \delta_{i j}+\frac{1+v}{E} \sigma_{i j}-\frac{v}{E} \delta_{i j} \sigma_{k k} \quad \text { with } i, j, k=r, \theta, \phi,
$$

where $g$ is the growing factor, $\delta_{i j}$ is Kronecker's delta, $\gamma_{r}, \gamma_{\theta} \in \mathbb{R}_{+}$, and $\gamma_{r}+2 \gamma_{\theta}=1$. Parameters $\gamma_{r}$ and $\gamma_{\theta}$ represent the proportions of the tumor growth in the radial and transversal directions, respectively.

Assuming small deformations, $\boldsymbol{e}=\frac{1}{2}\left(\nabla \boldsymbol{u}+(\nabla \boldsymbol{u})^{T}\right)$, using the material incompressibility (i.e., $v=\frac{1}{2}$ ) from hypothesis (iv), and applying a Jaumann derivative in (3), we obtain the relationship between the rates of deformation and stress

$$
\frac{1}{2}\left(\nabla \boldsymbol{v}+(\nabla \boldsymbol{v})^{T}\right)_{i j}=(\nabla \cdot \boldsymbol{v})\left(\delta_{1 i} \gamma_{r}+\left(\delta_{2 i}+\delta_{3 i}\right) \gamma_{\theta}\right) \delta_{i j}+\frac{1}{2 E} \frac{D}{D t}(3 \boldsymbol{\sigma}-\boldsymbol{I} \operatorname{tr}(\boldsymbol{\sigma}))_{i j} .
$$

3.3. External medium. From assumption (viii), the external medium satisfies the generalized Hooke's law

$$
\sigma_{i j}^{e}=\frac{E v}{(1+v)(1-2 v)} \delta_{i j} e_{k k}^{e}+\frac{E}{1+v} e_{i j}^{e},
$$

since the material is incompressible, $v=\frac{1}{2}$, and (5) becomes

$$
\sigma_{i j}^{e}=-p \delta_{i j}+\frac{2 E}{3} e_{i j}^{e}
$$

where $p$ is the isotropic pressure. 
3.4. Growth equation. For a living tissue, growth can be interpreted as the difference between cell production and cellular death. Then, from the mass-conservation continuity equation and hypothesis (v), we have

$$
\underbrace{\frac{\partial \rho}{\partial t}+\nabla \cdot(\boldsymbol{v} \rho)}_{\text {growth }}=\underbrace{\alpha c \rho\left(1+\eta_{1} \operatorname{tr}(\boldsymbol{\sigma})\right)}_{\text {cellular proliferation }}-\underbrace{k \rho\left(1-\eta_{2} \operatorname{tr}(\boldsymbol{\sigma})\right)}_{\text {cellular death }},
$$

where $\eta_{1}, \eta_{2} \in \mathbb{R}_{+}$are constants representing the dependence of cellular proliferation and death on stress.

As a consequence of tumor incompressibility (assumption (vi)), we get from (7)

$$
\nabla \cdot \boldsymbol{v}=\alpha c\left(1+\eta_{1} \operatorname{tr}(\boldsymbol{\sigma})\right)-k\left(1-\eta_{2} \operatorname{tr}(\boldsymbol{\sigma})\right) .
$$

3.5. Nutrient concentration. The nutrient concentration variation is determined by nutrient diffusion through the boundary of the tumor and its consumption by tumor cells in the interior. From assumptions (iv) and (v), it is noticed that

$$
\frac{\partial c}{\partial t}+\boldsymbol{v} \cdot \nabla c=\underbrace{D_{c} \nabla^{2} c}_{\text {diffusion }}-\underbrace{A_{c} c \rho}_{\text {consumption }}
$$

where $c$ represents the nutrient concentration, $D_{c}$ is the diffusion rate (which is assumed to be constant), and $A_{c}$ is the nutrient consumption rate. Moreover, assuming that the nutrient concentration variation is much smaller than its diffusion and consumption, (9) can be written as

$$
D_{c} \nabla^{2} c=A_{c} c \rho .
$$

3.6. Nondimensionalization of the model. An important step in modeling is to work with nondimensional variables. With this purpose, we define the constants

$$
L \equiv \sqrt{\frac{D_{c}}{A_{c} \rho}}, \quad \tau \equiv \frac{1}{\alpha c_{b}}, \quad c_{b}, \quad \text { and } \quad \epsilon \equiv \frac{k}{\alpha c_{b}},
$$

which represent the length scale, time scale, constant nutrient concentration at the boundary, and ratio between the cellular death and cellular proliferation rates. We use asterisks to identify the nondimensional variables:

$$
r^{*}=\frac{r}{L}, \quad \sigma_{i j}^{*}=\frac{\sigma_{i j}}{E}, \quad p^{*}=\frac{p}{E}, \quad v^{*}=\frac{v}{\alpha c_{b} L}, \quad t^{*}=\frac{t}{\tau}, \quad \text { and } \quad c^{*}=\frac{c}{c_{b}} .
$$

These new variables are placed in (1), (2), (4), (6), (8), and (10). For simplicity of notation, the asterisks are removed.

The equation of nutrients, derived from (10), can be solved analytically. We thus get

$$
c(r, t)=\frac{R \sinh r}{r \sinh R}
$$


Then taking into account the radial symmetry $\boldsymbol{v}=\left(v_{r}, 0,0\right)$ and placing (11) in the equation resulting from the nondimensionalization of (8) leads to

$$
\begin{aligned}
\frac{\partial v_{r}}{\partial r}(r, t)=\frac{R \sinh (r)}{r \sinh (R)}\left[1+\eta_{1} E\left(3 \sigma_{r}(r, t)-2 \beta(r, t)\right)\right] \\
-\epsilon\left[1-\eta_{2} E\left(3 \sigma_{r}(r, t)-2 \beta(r, t)\right)\right]-2 \frac{v_{r}(r, t)}{r} .
\end{aligned}
$$

Now, in matrix form, (4) reads

$$
\begin{aligned}
\left(\begin{array}{ccc}
\frac{\partial v_{r}}{\partial r} & 0 & 0 \\
0 & \frac{v_{r}}{r} & 0 \\
0 & 0 & \frac{v_{r}}{r}
\end{array}\right)=\frac{1}{r^{2}} \frac{\partial}{\partial r}\left(r^{2} v_{r}\right)\left(\begin{array}{ccc}
\gamma_{r} & 0 & 0 \\
0 & \gamma_{\theta} & 0 \\
0 & 0 & \gamma_{\theta}
\end{array}\right) \\
+\frac{1}{2 E}\left(\frac{\partial}{\partial t}+v_{r} \frac{\partial}{\partial r}\right)\left(\begin{array}{ccc}
\sigma_{1} & 0 & 0 \\
0 & \sigma_{2} & 0 \\
0 & 0 & \sigma_{3}
\end{array}\right)
\end{aligned}
$$

with

$$
\begin{aligned}
& \sigma_{1}=2 \sigma_{r}-\sigma_{\theta}-\sigma_{\phi}, \\
& \sigma_{2}=2 \sigma_{\theta}-\sigma_{r}-\sigma_{\phi}, \\
& \sigma_{3}=2 \sigma_{\phi}-\sigma_{r}-\sigma_{\theta},
\end{aligned}
$$

where $\sigma_{r}, \sigma_{\theta}$, and $\sigma_{\phi}$ are the diagonal components of the Cauchy stress tensor $\sigma$. In (13) the diagonal elements are the only nonzero elements because of the symmetry assumptions. If the second diagonal element is subtracted from the third in the matrix equation, then

$$
\left(\frac{\partial}{\partial t}+v_{r} \frac{\partial}{\partial r}\right)\left(\sigma_{\theta}-\sigma_{\phi}\right)=0
$$

suggesting $\sigma_{\theta}=\sigma_{\phi}$ because the material derivative of $\sigma_{\theta}-\sigma_{\phi}$ is zero. Substituting this result in the first diagonal element of (13), we have

$$
\left(\frac{\partial}{\partial t}+v_{r} \frac{\partial}{\partial r}\right)\left(\sigma_{r}-\sigma_{\theta}\right)=2\left(\gamma_{\theta} \frac{\partial v_{r}}{\partial r}-\gamma_{r} \frac{v_{r}}{r}\right)
$$

As in (4), the equilibrium (2) represents a system of partial differential equations, i.e., three partial differential equations for three unknown functions $\left(\sigma_{r}, \sigma_{\theta}\right.$, and $\left.\sigma_{\phi}\right)$, and the only nonzero equality is that corresponding to the radial direction, i.e.,

$$
\frac{\partial \sigma_{r}}{\partial r}+\frac{2\left(\sigma_{r}-\sigma_{\theta}\right)}{r}=0
$$


Finally, from (1), (12), (14), and (15), the model is given by the system of firstorder partial differential equations

$$
\begin{aligned}
\frac{d R}{d t}(t)=v_{r}(R, t) & \text { for } t \in \mathbb{R}_{+}^{*}, \\
\frac{\partial v_{r}}{\partial r}(r, t)=\frac{R \sinh (r)}{r \sinh (R)}\left[1+\eta_{1} E\left(3 \sigma_{r}(r, t)-2 \beta(r, t)\right)\right] & \\
-\epsilon\left[1-\eta_{2} E\left(3 \sigma_{r}(r, t)-2 \beta(r, t)\right)\right] & \text { for } t \in \mathbb{R}_{+}^{*} \text { and } r \in(0, R], \\
-2 \frac{v_{r}(r, t)}{r} & \text { for } t \in \mathbb{R}_{+}^{*} \text { and } r \in[0, R), \\
\frac{\partial \sigma_{r}}{\partial r}(r, t)=-\frac{2 \beta}{r} & \text { for } t \in \mathbb{R}_{+}^{*} \text { and } r \in(0, R),
\end{aligned}
$$

with

$$
\begin{aligned}
& \varpi(r, t)=2\left(\gamma_{\theta} \frac{\partial v_{r}}{\partial r}-\gamma_{r} \frac{v_{r}}{r}\right) \\
&=2 \gamma_{\theta}\left(\frac{R \sinh (r)}{r \sinh (R)}\left[1+\eta_{1} E\left(3 \sigma_{r}(r, t)-2 \beta(r, t)\right)\right]\right. \\
&\left.\quad-\epsilon\left[1-\eta_{2} E\left(3 \sigma_{r}(r, t)-2 \beta(r, t)\right)\right]\right)-2 \frac{v_{r}(r, t)}{r},
\end{aligned}
$$

subject to the initial and boundary conditions

$$
\begin{aligned}
R(0) & =R_{0}, \\
\sigma_{r}(r, 0) & =0, \\
\beta(r, 0) & =0, \\
v(0, t) & =0,
\end{aligned}
$$

where $\beta=\sigma_{r}-\sigma_{\theta}$. Two conditions are still needed for $\beta$ and $\sigma_{r}$.

(i) Condition for $\beta$ at $r=0$. Since $v_{r}=0$ at $r=0$ and the derivatives of $\beta$ are bounded (because $\beta$ is assumed $C^{1}$ in $[0, R]$ with respect to $r$ ), then

$$
\frac{\partial \beta}{\partial t}(0, t)=\varpi(0, t) .
$$

Therefore, the first boundary condition for $\beta$ is

$$
\begin{aligned}
\frac{\partial \beta}{\partial t}(0, t)=\left(2 \gamma_{\theta}-1\right)\left(\frac{R}{\sinh (R)}[\right. & \left.1-\eta_{1} E\left(3 \sigma_{r}(0, t)-2 \beta(0, t)\right)\right] \\
& \left.-\epsilon\left[1+\eta_{2} E\left(3 \sigma_{r}(0, t)-2 \beta(0, t)\right)\right]\right)+\frac{\partial v_{r}}{\partial r}(0, t) .
\end{aligned}
$$


(ii) Condition for $\sigma_{r}$. This condition may be determined if the constitutive equation (6) of the external medium is used assuming the continuity of the stresses at the tumor boundary. Because of the spherical tumor symmetry from hypothesis (iii) and the incompressibility of the external medium (hypothesis (viii)),

$$
\operatorname{tr}(\boldsymbol{e})=\frac{1}{r^{2}} \frac{\partial}{\partial r}\left(r^{2} u_{r}\right)=0 .
$$

Substituting the solution of the above equation in (6), we have

$$
\left.\sigma_{r}\right|_{r=R}=-\frac{4\left(R-R_{0}\right)}{3 R} \text {. }
$$

\section{Cellular automata model definition}

A cellular automaton based on a linear elasticity tumor model [Ramírez-Torres et al. 2016] is defined. The model described in [Ramírez-Torres et al. 2016] and summarized in the previous section is taken as an example of a system of differential equations (DE) that represents tissue growth, i.e., tumor growth in this case.

The present continuum-mechanical model considers the tumor as a solid in the three-dimensional space, and the forces on it are considered acting per volume unit. As a result of the spherical symmetry hypothesis, the body deformation is the same in two of the three principal directions and no shear deformations are accounted for. Moreover, from the symmetry condition, all the fields depend only on time $t$ and on one spatial variable $r$. The three principal stresses are $\sigma_{r}, \sigma_{\theta}$, and $\sigma_{\phi}$, with (as shown in the text after (13)) $\sigma_{\theta}=\sigma_{\phi}$. In this sense, we only need to find $\sigma_{r}$ and $\sigma_{\theta}$, given that the stress in the direction orthogonal to the plane where they are contained, corresponding to the third principal stress, is equal to $\sigma_{\theta}$ and therefore not necessarily zero. Hence, the theoretical model is addressed as neither a plane strain nor as a plane stress problem [Sokolnikoff 1956].

On the other hand, for representing the cellular automaton model, a cross section passing through the origin of the tumor in the three-dimensional model is taken. It does not matter which cross section because, given the spherical symmetry of the continuous theoretical model, all the cross sections are equivalent. Therefore, the cellular automaton is constructed in a two-dimensional setting, although the model is easily extended to three dimensions. Indeed, the rules of the model are given for any spatial dimension. Hence, the stresses and deformations in the cellular automaton model are the same stresses and deformations which are completely contained in a cross section of the continuum-mechanical model.

The transformation of the deterministic DE linear elasticity tumor model into a cellular automaton is required. The neighborhood structure and the rules for a probabilistic CA are deduced from continuous differential equations. The CA solution presented in this work can be extended to other similar continuous DE models. 
4.1. General model definition. In the present study, a simple two-state cellular automaton is considered, where a 0 -state represents a normal cell and a 1-state exemplifies a tumoral cell.

The automaton is considered infinite because it is defined in a borderless square lattice in which there exists some central cell with coordinates $(0,0)$. The initial state assigned to each cell is 0 , except for a finite number of them. Practical implementations of this model must maintain a finite matrix representation of the relevant part of the lattice and expand it on demand.

The neighborhood structure and a rule set that describes the continuous model behavior are explained in the following.

4.2. Neighborhood structure selection. There is an equivalence (1) between the tumor radius growth speed and the tumor cell displacement speed in the continuous model described in [Ramírez-Torres et al. 2016]:

$$
\frac{d R}{d t}=v(R, t)
$$

The speed $v$ could be used in the definition of the transition rules of the CA because the "displacement speed" oriented outside the tumor is closely related to the tumor cell propagation. In addition, we know that $v$ does not depend explicitly on the spatial coordinates of a point but instead on the radial one. This fact implies that the "influence" of a tumor is equally distributed in all directions from the center of the model.

In order to select the appropriate neighborhood structure, we should take into account this property of the tumor growth speed. Since all the discrete time steps are equal, the increments of the tumor cell coordinates should also be equal, for the same moment of time, in all directions. In the CA, the increment of the tumor cell coordinate is described as the propagation of some tumor cell into another normal cell.

Therefore, the influence zone (neighbor) of any cell $c$ must have cells with the same distance to $c$. We can choose some constant distance $q$, and for any cell $c_{0}$, the neighborhood is defined as

$$
\left\{c \in C: d\left(c_{0}, c\right)=q\right\},
$$

where $d\left(c_{1}, c_{2}\right)$ is the common Euclidean distance in the unit square lattice.

We choose $q=1$, which generates a well known von Neumann neighborhood [Deutsch and Dormann 2005]. It is possible to choose other values of $q$, such as $q=\sqrt{2}$ or $q=2$, generating other unexplored neighborhoods.

4.3. Rule inference. The main principle used in the area of rule inference from continuous models [Guinot 2002] consists of creating a stochastic rule with the 
structure

$$
s_{c}(t+\Delta t)= \begin{cases}s_{c}(t)+1, & X \leq g\left(s_{c}(t), N(c), \Delta t\right), \\ s_{c}(t), & X>g\left(s_{c}(t), N(c), \Delta t\right),\end{cases}
$$

where $X$ is a random variable with uniform distribution in $(0,1)$ and $\Delta t$ is the length of the time step. The hardest part of rule inference is precisely the correct and meaningful definition of $g$. Actually, function $g$ expresses the idea of the "speed" of growing in some place of the lattice where conditions around the cell are expressed by the state of the cell itself $s_{c}(t)$ and by its neighborhood $N(c)$.

From the continuous model, the differential equation (1) shows the relationship between the tumor radius growth speed and the radius itself:

$$
\frac{d R}{d t}=v(R, t)
$$

Initially, we assume that $v(R, t) \geq 0$, i.e., the size of the tumor is nondecreasing. We interpret $\frac{d R}{d t}$ as the average number of new tumor cells created from one tumor cell per unit of time. If $\frac{d R}{d t}<1$, this speed is seen as the probability of appearance of a new tumor cell during the unit time step, i.e., function $g$. Otherwise, some normalization process over $\frac{d R}{d t}$ becomes necessary.

In the case when $\eta_{1}=\eta_{2}=0$ (stress dependence is not considered), a closedform expression for $v$ can be obtained from (16)-(19):

$$
\frac{d R}{d t}=\frac{\cosh R}{\sinh R}-\frac{1}{R}-\frac{\epsilon R}{3},
$$

where $\epsilon$ is a constant. The fact that $v(R, t)=v(R)$, depending only on the maximum tumor radius, is particularly important for the rule definition process because cellular automaton inherently does not have any notion of time: the next state depends only on the current state.

However, if $\eta_{1} \neq 0$ or $\eta_{2} \neq 0$, then the stress influence is taken into account and no closed-form expression can be obtained for $v$. In this case, $v(R, t)$ is approximated using numerical methods, like Euler's method or the Lax-Wendroff scheme from finite differences.

4.3.1. Case without stress dependence. If $\eta_{1}=\eta_{2}=0$, stresses are not taken into account, and a closed-form expression (21) for $v$ is obtained from the differential equations (16)-(19). The properties of this equation are analyzed in order to appropriately define $g$.

Stability analysis. Equation (21) has the form $R_{t}^{\prime}=v(R)$, where $R_{t}^{\prime}$ is the derivative of the radius function with respect to time. In the classical numerical methods, we can use the fact that, for a small $h$,

$$
R_{t}^{\prime} \approx \frac{R_{t+1}-R_{t}}{h} \quad \text { and } \quad R_{t+1} \approx R_{t}+h v(R) .
$$




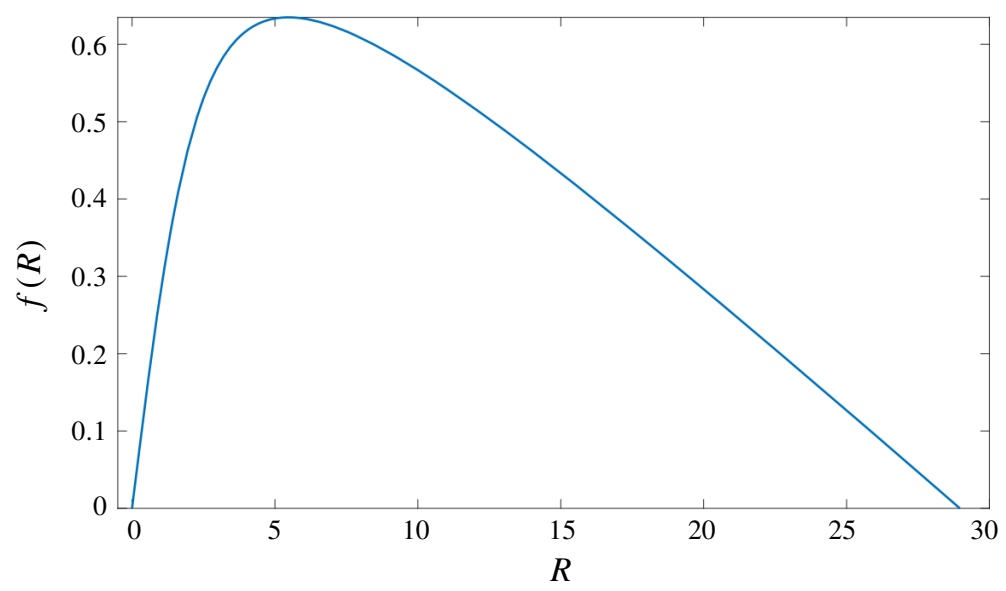

Figure 1. Case without stress dependence, stability analysis: increment function $(\cosh R) /(\sinh R)-1 / R-\epsilon R / 3$ for $\epsilon=0.1$.

It is clear that, for a discrete time step $h, v(R)$ represents the increment in the tumor radius from a time moment to the next. In terms of cellular automata, it represents the magnitude of propagation of a tumoral cell $c$ into its normal neighbor. However, since CA are discrete computational systems in space, the tumoral cell cannot be expanded in a fractional amount of space.

In Figure 1, the increment $v(R)$ reaches a local maximum close to $v=0.63$ and has a root close to $R=29$. The tumor radius starts its growth at $R=R_{0} \geq 0$. Since the increment is always positive, it grows asymptotically to the root value, where the increment is null.

We analyze the rule inference process, where the function $g$ is appropriately defined. In rule (20), $g$ indicates the probability of expansion of a tumoral cell into a normal neighbor. In this sense, the desired expansion probability is exactly the magnitude of propagation of the tumor cell. Since the increment $v(R) \in[0,1]$ is bounded, it can be used as the probability $g$, simply setting $g=v$. If $v(R) \notin[0,1]$, it would have been necessary to perform some normalization process over $v(R)$.

To summarize, if the stress dependence is not considered, the following stochastic rule emerges:

$$
s_{c}(t+\Delta t)= \begin{cases}s_{c}(t)+1, & X \leq v(r), \\ s_{c}(t), & X>v(r),\end{cases}
$$

where $X$ is a random variable with uniform distribution in $(0,1)$,

$$
v(r)=\frac{\cosh r}{\sinh r}-\frac{1}{r}-\frac{\epsilon r}{3},
$$


and $r=\sqrt{i^{2}+j^{2}}$ is the radial coordinate of a cell. Although the angular coordinate is not present in this rule, this does not mean that the model is symmetric in all polar directions. The asymmetry is present indirectly through random variable $X$ whose realizations must be generated at every time step and for each cell [Guinot 2002], leading to an irregular shape.

4.3.2. Case with stress dependence. The assumption that $\eta_{1} \neq 0$ or $\eta_{2} \neq 0$ leads to a scenario where the stresses' influence is considered. In particular, no closedform expression can be obtained for $v$ in this case, making the use of numerical methods unavoidable. Therefore, $v$ is approximated using Euler's method or the Lax-Wendroff scheme from finite differences [Ramírez-Torres et al. 2016]. More precisely, numerical approximations of $R(t)$ and $v(R, t)$ are obtained. This means that pairs $\left(R_{i}, v\left(R_{i}\right)\right)$ are computed for each moment in time $t_{i}$, where

- $R_{i}$ is the tumor radius at $t_{i}$ and

- $v\left(R_{i}\right)$ is the tumor growth speed at any point of the tumor border at $t_{i}$.

There is no guarantee of the existence of a functional dependency between $v$ and $R$, as in (21). In fact, there are cases where for the same value of $R$, more values of $v$ are obtained. One example is shown in Figure 2, where no $v(R)$ can be defined because $R(t)$ is not injective, with two distinct growth speeds at the same radius, at two distinct moments of time.

We first analyze the case where the function $v(R)$ is approximately definable. Next, we examine, the case where the function $v(R)$ cannot be defined.

(i) Definable $v(R)$. If $R(t)$ is a strictly increasing function, then it is also injective and an ordered sequence of pairs $\left(R_{i}, v\left(R_{i}\right)\right)$ is obtained, where all radius values

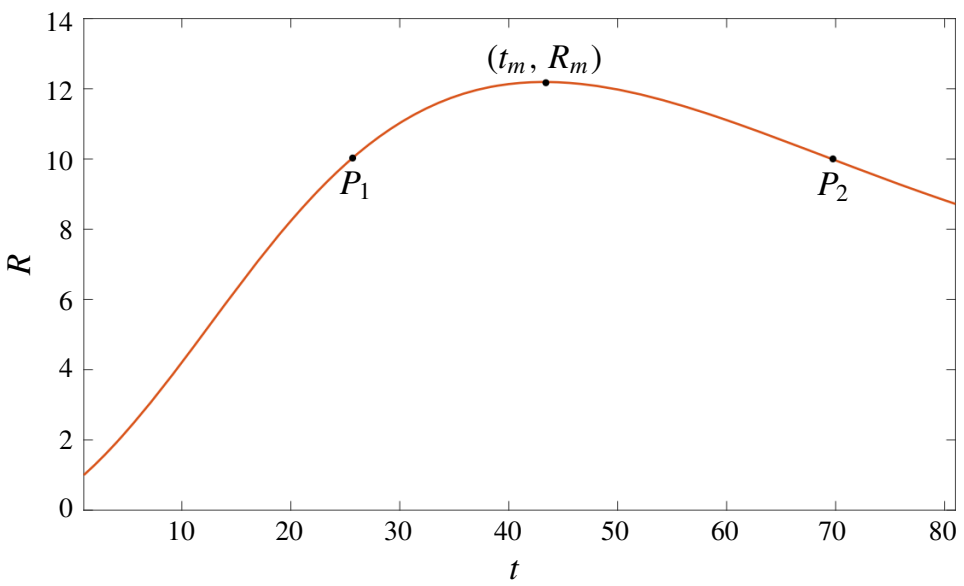

Figure 2. Tumor radius growth in time, theoretical model, strict extremum case. 
are different. In this case, function $v(R)$ is approximately defined in the following way. To evaluate $v(R)$ for some $R=R_{j}$, the speed value that corresponds to the closest to $R_{j}$ among all the radius values in the sequence is selected.

The smaller the time step becomes, the better the approximation is, since there is a higher density of available $R$ values.

Given this definition of $v(R)$, the stochastic rule for the CA is defined in a similar way as in the case without stress dependence (22):

$$
s_{c}(t+\Delta t)= \begin{cases}s_{c}(t)+1, & X \leq v\left(R_{i}\right), \\ s_{c}(t), & X>v\left(R_{i}\right) .\end{cases}
$$

Here, $v\left(R_{i}\right)$ is the evaluation of the radius $R_{i}$ that is closest to the radial coordinate of the cell value, from the available radius-speed evaluations.

The sequence of pairs $\left(R_{i}, v\left(R_{i}\right)\right)$ is called from now on the guide set and represents an approximation to the actual $v$ function. It is used as a guide during the CA evolution process.

(ii) Undefinable $v(R)$. If $R(t)$ is not a strictly increasing (or strictly decreasing ${ }^{1}$ ) function, the injectivity of $R(t)$ cannot be guaranteed and no simple functional dependency between $v$ and $R$ can be defined.

In particular, the special case where $R(t)$ has a strict extremum is considered. In Figure 2 an example is shown. The existence of some pair $\left(R_{m}, v\left(R_{m}\right)\right)$ is assumed, where $R_{m}$ is a maximum over the $R$ values available in the guide set.

The impossibility of defining a single CA that represents the continuous model behavior is explained below. Choosing two time moments with the same tumor radius (points $P_{1}$ and $P_{2}$ in Figure 2), similar CA configurations are expected in both cases. Then since the cellular automaton is a memoryless system (no time variable or history can be present), it is not possible to distinguish between both states, in order to determine when it should grow or when a reduction should take place, since the next state of any CA only depends on its previous state.

In response to the need of having the tumor growth process representation in this case, a solution based on the use of two cellular automata is proposed. Having a sequence of the $\left(R_{i}, v\left(R_{i}\right)\right)$ pairs ordered by $i$, the sequence is divided into two subsequences. The first is the sequence $s_{1}$ that starts with $\left(R_{0}, v\left(R_{0}\right)\right)$ and ends with $\left(R_{m}, v\left(R_{m}\right)\right)$; the second sequence $s_{2}$ contains the rest of the radius-speed pairs up to the last (see Figure 2).

It was shown that a guide set like $s_{1}$ can define an approximation to the positive $v$ function needed to define the rule (23). Another rule that reflects tumor reduction

${ }^{1}$ The assumption that $v(R, t) \geq 0$ is relaxed here; i.e., the tumor may decrease with negative values of $v$. 
is then required. In this sense, the following rule structure is proposed:

$$
s_{c}(t+\Delta t)= \begin{cases}s_{c}(t)-1, & X \leq\left|v\left(R_{i}\right)\right|, \\ s_{c}(t), & X>\left|v\left(R_{i}\right)\right| .\end{cases}
$$

This rule is applied to the tumoral cells with normal neighbors, where $X$ is a random variable with uniform distribution in $(0,1)$ and $v\left(R_{i}\right)$ is the evaluation of the radius $R_{i}$ from $s_{2}$ that is closest to the radial coordinate of the cell $r$.

Finally, the complete model is defined as an ordered pair $\left(A_{1}, A_{2}\right)$, where:

- $A_{1}$ corresponds to the first of the two automata, with stochastic rule (23), and guide set $s_{1}$ and

- $A_{2}$ corresponds to the second automaton, with its initial state being the final state of $A_{1}$, stochastic rule (24), and guide set $s_{2}$.

4.3.3. Rule definition summary. To summarize, a new stochastic model of tumor growth obtained from a differential equation system is provided. A random variable $X$ with uniform distribution in $(0,1)$ is considered. If a closed-form expression of the growth speed $v$ in the tumor border can be found, then the following rule is defined:

$$
s_{c}(t+\Delta t)= \begin{cases}s_{c}(t)+1, & X \leq v(r) \\ s_{c}(t), & X>v(r)\end{cases}
$$

where $v(r)=(\cosh r) /(\sinh r)-1 / r-\epsilon r / 3$ and $r=\sqrt{i^{2}+j^{2}}$ is the radial coordinate of the cell $c$ in the lattice.

If a closed-form expression for $v$ cannot be found, then a guide set is defined as a collection of pairs $\left(R_{i}, v\left(R_{i}\right)\right)$ ordered by the time moment at which the approximation is made. If $v \geq 0$, then the following first CA rule can be applied to the normal cells with at least one tumoral cell in its neighborhood:

$$
s_{c}(t+\Delta t)= \begin{cases}s_{c}(t)+1, & X \leq v\left(R_{i}\right), \\ s_{c}(t), & X>v\left(R_{i}\right),\end{cases}
$$

where $v\left(R_{i}\right)$ is the evaluation of $v$ at the radius $R_{i}$ from the guide set that is closest to the radial coordinate of the cell $r$. We remark that this rule is applied to the cells with tumoral neighbors and, in particular, to the cells on the border of the tumor, where $r \approx R$.

If $v<0$, then a second CA rule is applied to the tumoral cells with normal neighbors:

$$
s_{c}(t+\Delta t)= \begin{cases}s_{c}(t)-1, & X \leq\left|v\left(R_{i}\right)\right|, \\ s_{c}(t), & X>\left|v\left(R_{i}\right)\right|,\end{cases}
$$

where, once again, $v\left(R_{i}\right)$ is the evaluation of $v$ from the guide set at the closest point to the radial coordinate of the cell $r$. 


\section{Results}

One important issue in any model is the validation of its consistency with the original phenomenon or another reference model. In this case, a comparison between the behavior of the discrete CA model described above and the continuous model is provided.

In this section, experimental results are discussed for all three cases of tumor growth: without stress dependence, with stress dependence and strictly increasing tumor radius, and with stress dependence and a tumor radius that has a strict extremum.

The CA model described above was implemented using the $\mathrm{C} \#$ programming language on an Intel Core i 7 machine, with a $2.0 \mathrm{GHz}$ processor and $16 \mathrm{~GB}$ of random-access memory. All executions were very fast, taking a few seconds of running time.

Common values of the parameters $\epsilon=0.1, R_{0}=1$, and $E=64.0439$ were fixed in all computations. An important clarification is that the tumor radius value in the cellular automaton is computed as the average between the tumoral border cell distances to the center of the tumor.

5.1. Tumor growth without stress dependence. In the case where $\eta_{1}=\eta_{2}=0$, the stresses are not taken into account in the mathematical model. Then the cellular automaton with rule (22) behavior is compared with the continuous differential equation model. The results are shown in Figure 3.

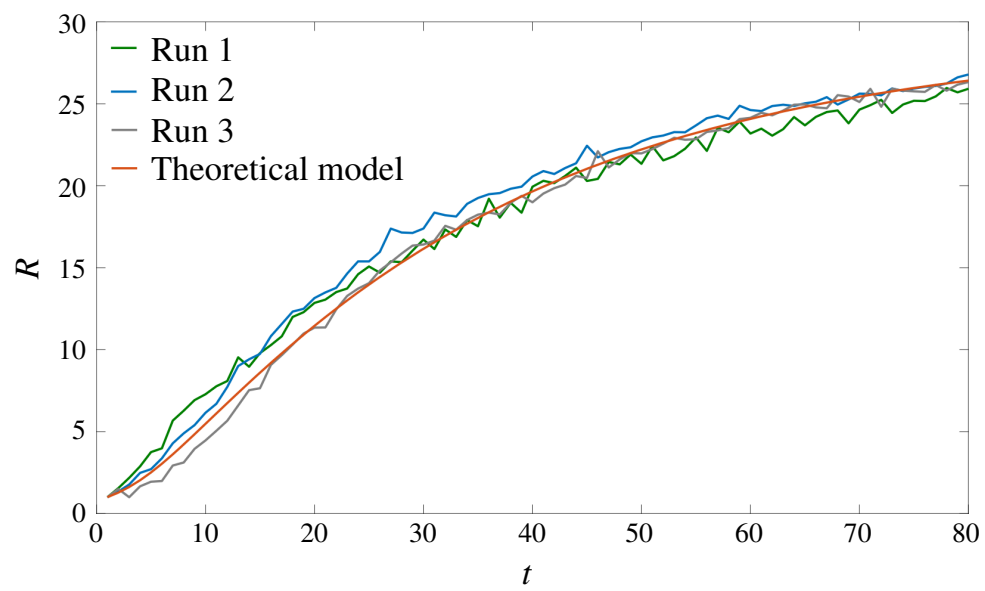

Figure 3. Graphic illustrating the evolution of the tumor radius with time, case without stress dependence. Comparison between several runs of the discrete CA model and the continuous (red line) model. The continuous model is obtained from (21), as a particular case of the model defined by (16)-(19). 

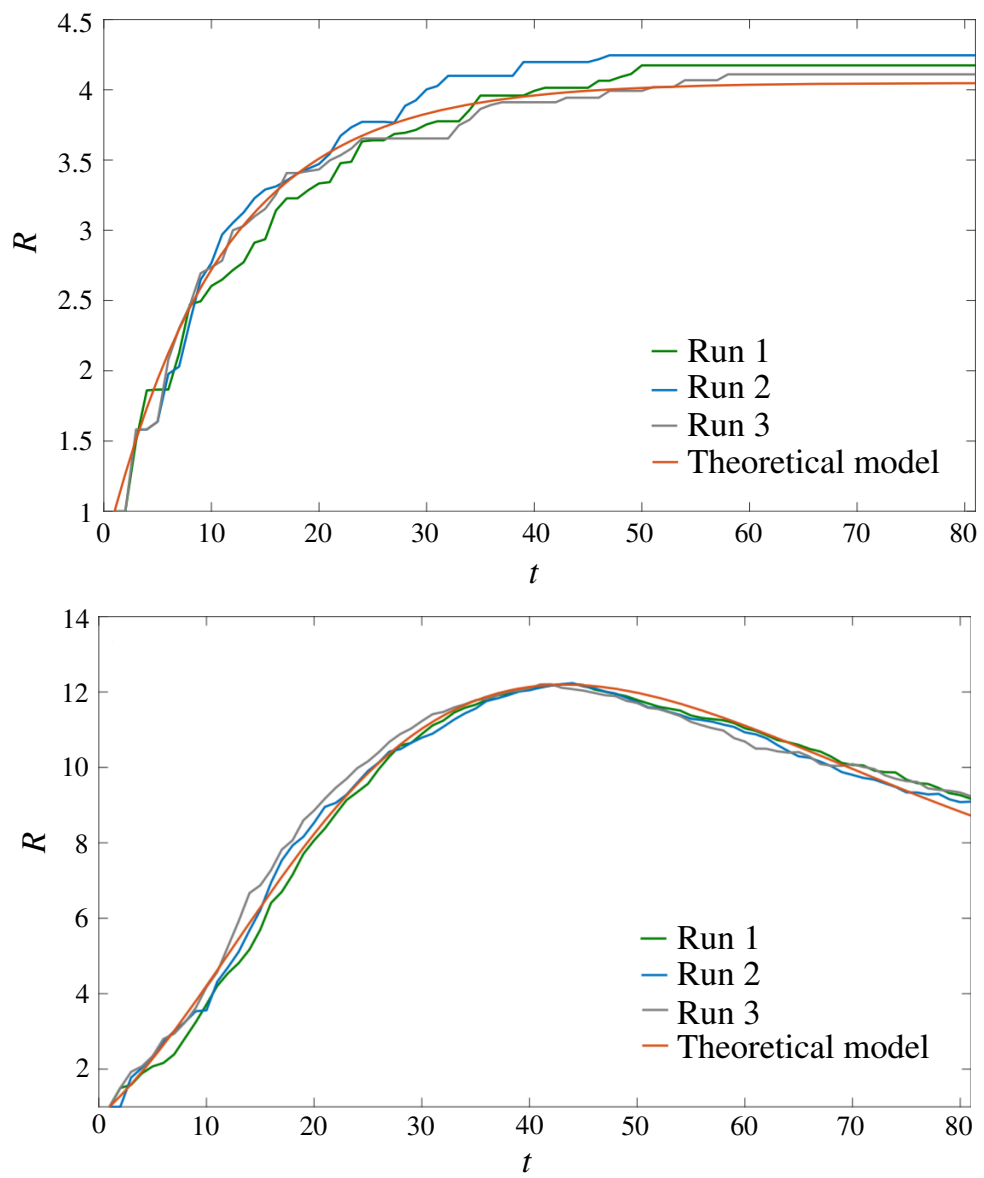

Figure 4. Graphics illustrating the evolution of the tumor radius with time, cases with stress dependence when the tumor radius is strictly increasing (top) and when the tumor radius has a strict extremum (bottom). Comparison between several runs of the discrete CA model and the continuous (red line) model.

The radius-time dependence graphics are similar and close to each other. A small difference between them is due to the dissimilar nature of the models.

5.2. Tumor growth with stress dependence. Similar experiments are performed in the case of stress-dependent tumor growth. Results are shown in Figure 4.

The case where the tumor radius is a strictly increasing function is obtained with $\gamma_{\theta}=\frac{1}{3}$ and $\eta_{1}=0.004$ (Figure 4, top). A cellular automaton with rule (23) is used.

The case where the tumor radius has a strict extremum is obtained with $\gamma_{\theta}=\frac{1}{10}$ and $\eta_{1}=0.002$ (Figure 4, bottom). In this case, two cellular automata with rules (23) and (24), respectively, are used. 

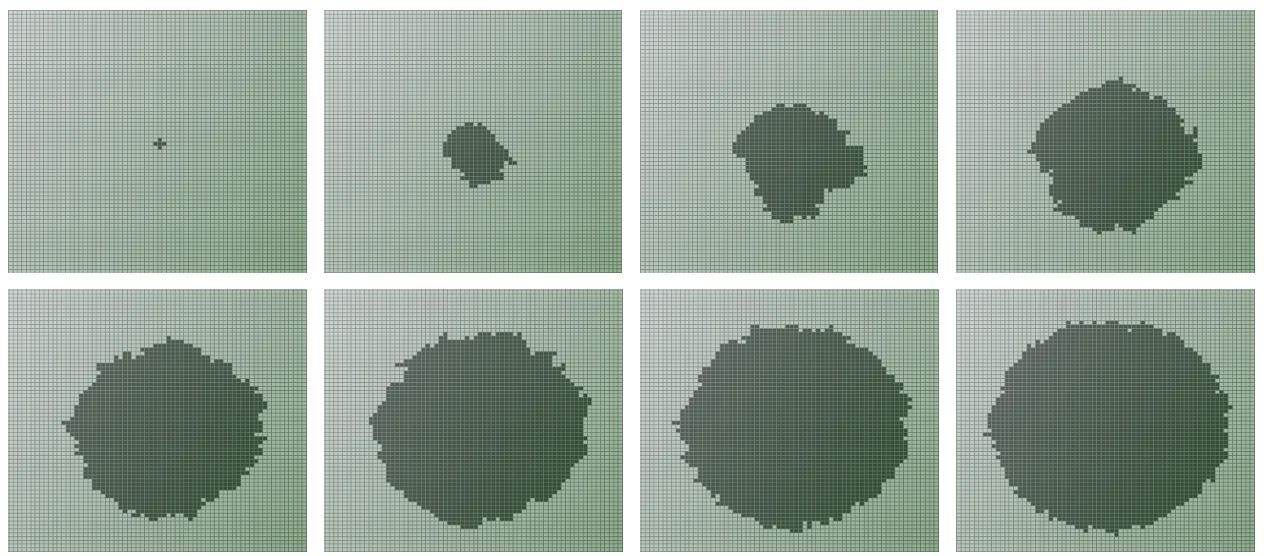

Figure 5. Visualizations of the CA tumor growth for time moments $0,10,20,30,40,50,60$, and 70, respectively.

In both cases, close radius-time dependence graphics are observed. Stabilization of tumor radius growth is detected in the strictly increasing function case (Figure 4, top) in all runs. An excellent degree of proximity is achieved in the strict extremum case (Figure 4, bottom) with two cellular automata.

5.3. Tumor growth visualization in time. An example of the tumor growth visualization is shown in Figure 5. It corresponds to the case without stress dependence described in Section 5.1.

In particular, we notice an exponential, fast growth at the beginning, followed by an asymptotic behavior when the radius of the tumor stabilizes around a constant value. Further visualizations are not shown due to their similarity with those corresponding to $t=60$ and $t=70$. There is almost no change when the running time is increased because of the strong convergence of the tumor radius in the CA model.

This corresponds to the stability results obtained in Section 4.3.1 and shown in Figure 1, where the radius increment function has a zero at a radius value slightly below 29.

5.4. Dispersion of the executions. Finally, the dispersion of computational executions is evaluated. As an example, the case without stress dependence is considered, whose visualizations were shown in the previous section. Firstly, the average execution is computed as a sequence of averages of tumor radius values obtained from $100 \mathrm{CA}$ model runs, for each time step. The resulting graphic is shown in Figure 6. It compares the CA average execution with the continuous model: both functions are very close to each other.

Figure 7 displays the coefficient of variation. Most of the chart is below the 


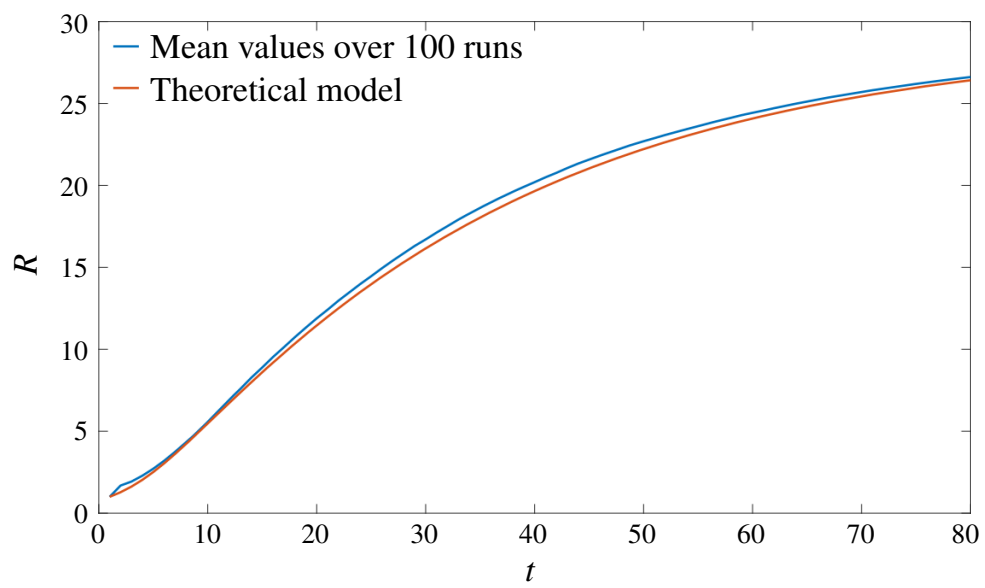

Figure 6. The blue line shows average values of the tumor radius variable over $100 \mathrm{CA}$ model runs. Each point is obtained as the average of 100 tumor radius values obtained at that moment of time by 100 independent executions of the cellular automaton model. The red line shows the continuous model obtained from (21) as a particular case of the model defined by (16)-(19).

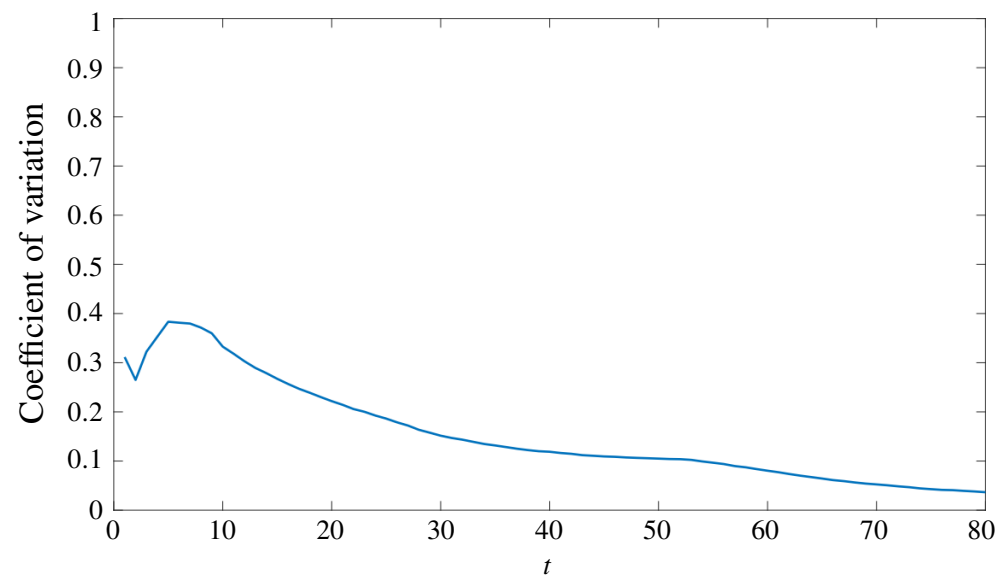

Figure 7. Values (in percent) of the coefficient of variation of the tumor radius variable for the CA model. Each point is obtained from 100 tumor radius values reached at that moment of time by 100 independent executions of the CA model.

$20 \%$ line, indicating small variations of the tumor radius. Larger variations are observed only near the start of the executions. This shows a stable behavior of the computational model beyond its stochastic nature. 


\section{Conclusions}

A new stochastic cellular automata model for the process of tumor growth is proposed. From a linear elasticity deterministic continuous tumor model described in [Ramírez-Torres et al. 2016], a neighborhood structure and stochastic automata rules are deduced. The results allow one to visualize the growth process described in the continuous model in a more realistic manner since tumors are neither regular nor perfectly circular. Moreover, stress influence in the growing of the tumor is taken into account at the time when the rules of the CA model are derived. The differences in the tumor radius between the two models are small and are actually due to their different nature. Validation tests confirmed that the CA model accurately captures the hypothesis of the described phenomena. The methodology exposed in this work can be applied to other continuous DE models in order to represent the growth processes in a nonidealized and nondeterministic way.

\section{Acknowledgments}

Ruben Interian has been sponsored by a CAPES scholarship. Ariel Ramírez-Torres thanks the postdoctoral funding of the Program of Postdoctoral Scholarships DGAPA from UNAM and professors Federico J. Sabina and Catherine García-Reimbert for their support. Work of Celso C. Ribeiro was partially supported by CNPq research grant 459575/2014-7 and by FAPERJ research grant E-26/201.198/2014. Aura Conci is partially supported by FAPERJ project SIADE2 and by CNPq project number 303240/2015-6.

\section{References}

[Anderson et al. 2009] A. R. A. Anderson, K. A. Rejniak, P. Gerlee, and V. Quaranta, "Microenvironment driven invasion: a multiscale multimodel investigation", J. Math. Biol. 58:4-5 (2009), $579-624$.

[Berto and Tagliabue 2012] F. Berto and J. Tagliabue, "Cellular automata", electronic reference, 2012, Available at http://plato.stanford.edu/entries/cellular-automata/.

[Boondirek et al. 2010] A. Boondirek, W. Triampo, and N. Nuttavut, "A review of cellular automata models of tumor growth", Int. Math. Forum 5:61 (2010), 3023-3029.

[Deutsch and Dormann 2005] A. Deutsch and S. Dormann, Cellular automaton modeling of biological pattern formation: characterization, applications, and analysis, Birkhäuser, 2005.

[Dormann and Deutsch 2002] S. Dormann and A. Deutsch, "Modeling of self-organized avascular tumor growth with a hybrid cellular automaton”, In Silico Biol. 2:3 (2002), 393-406.

[Guinot 2002] V. Guinot, "Modelling using stochastic, finite state cellular automata: rule inference from continuum models", Appl. Math. Model. 26:6 (2002), 701-714.

[Hoehme and Drasdo 2010] S. Hoehme and D. Drasdo, "A cell-based simulation software for multicellular systems”, Bioinformatics 26:20 (2010), 2641-2642. 
[Hu and Ruan 2003] R. Hu and X. Ruan, "A simple cellular automaton model for tumor-immunity system”, pp. 1031-1035 in IEEE International Conference on Robotics, Intelligent Systems and Signal Processing (Changsha, China, 2003), vol. 2, Inst. Electrical and Electronics Engrs., 2003.

[Kansal et al. 2000a] A. Kansal, S. Torquato, E. A. Chiocca, and T. S. Deisboeck, "Emergence of a subpopulation in a computational model of tumor growth", J. Theor. Biol. 207:3 (2000), 431-441.

[Kansal et al. 2000b] A. Kansal, S. Torquato, G. R. Harsh, IV, E. A. Chiocca, and T. S. Deisboeck, "Simulated brain tumor growth dynamics using a three-dimentional cellular automaton", J. Theor. Biol. 203:4 (2000), 367-382.

[Merks and Glazier 2005] R. M. H. Merks and J. A. Glazier, "A cell-centered approach to developmental biology", Physica A 352:1 (2005), 113-130.

[Ngwa and Agyingi 2012] M. Ngwa and E. Agyingi, "Effect of an external medium on tumor growthinduced stress", IAENG Int. J. Appl. Math. 42:4 (2012), 229-236.

[Ramírez-Torres et al. 2016] A. Ramírez-Torres, F. Valdés-Ravelo, R. Rodríguez-Ramos, J. BravoCastillero, R. Guinovart-Díaz, and F. J. Sabina, "Modeling avascular tumor growth via linear elasticity", pp. 1739-1740 in Contributions to the foundations of multidisciplinary research in mechanics (Montreal, 2016), vol. 3, edited by J. M. Floryan, Proc. Int. Congress Theoretical and Applied Mechanics 24, Int. Union Theoretical Applied Mechanics, 2016.

[Rejniak and Anderson 2011] K. A. Rejniak and A. R. A. Anderson, "Hybrid models of tumor growth", Wiley Interdiscip. Rev. Syst. Biol. Med. 3:1 (2011), 115-125.

[Rejniak and McCawley 2010] K. A. Rejniak and L. J. McCawley, "Current trends in mathematical modeling of tumor-microenvironment interactions: a survey of tools and applications", Exp. Biol. Med. 235:4 (2010), 411-423.

[Sokolnikoff 1956] I. S. Sokolnikoff, Mathematical theory of elasticity, 2nd ed., McGraw-Hill, 1956.

Received 4 Dec 2016. Revised 23 Apr 2017. Accepted 6 Jun 2017.

RUBEN INTERIAN: rubenus@yandex.ru

Institute of Computing, Fluminense Federal University, Niterói, Brazil

REINALDO RODRÍGUEZ-RAMOS: rerora2006@gmail.com

Mathematics and Computer Science Faculty, Havana University, Havana, Cuba

FERnANDo VALDÉS-RAVELO: fernandov@cim.sld.cu

Molecular Immunology Center, Havana, Cuba

ARIEL RAMÍREZ-TORRES: arielrt02@gmail.com

Institute for Research in Applied Mathematics and Systems, National Autonomous University of Mexico, Mexico City, Mexico

Celso C. Ribeiro: celso@ic.uff.br

Institute of Computing, Fluminense Federal University, Niterói, Brazil

AURA CONCI: conci.aura@gmail.com

Institute of Computing, Fluminense Federal University, Niterói, Brazil 
EDITORIAL BOARD

ANTONIO CARCATERRA

ERIC A. CARLEN

FRANCESCO DELL'ISOLA

RAFFAELE ESPOSITO

ALBERT FANNJIANG

Gilles A. FranCFORT

Pierangelo MARCATI

JEAN-JACQUES MARIGO

PETER A. MARKOWICH

MARTIN OSTOJA-STARZEWSKI

PIERRE SEPPECHER

DAVID J. STEIGMANN

PAUl STEINMANN

PierRe M. SuQueT

MANAGING EDITORS

MICOL AMAR

CORRADO LATTANZIO

ANGELA MADEO

MARTIN OSTOJA-STARZEWSKI

\section{ADVISORY BOARD}

ADNAN AKAY

Holm AltenBaCH

MICOL AMAR

HARM ASKES

TEODOR ATANACKOVIĆ

VICTOR BERDICHEVSKY

GUY BOUCHITTÉ

ANDREA BRAIDES

ROBERTO CAMASSA

MAURO CARFORE

ERIC DARVE

FELIX DARVE

ANNA DE MASI

GianPiEtro DEL Piero

EMMANUELE Di BENEDETTO

BERNOLD FIEDLER

IRENE M. GAMBA

DAVID Y. GAO

SERGEY GAVRILYUK

TIMOTHY J. HEALEY

DOMINIQUE JEULIN

ROGER E. KHAYAT

CORRADO LATTANZIO

ROBERT P. LIPTON

ANGELO LUONGO

ANGELA MADEO

JUAN J. MANFREDI

CARLO MARCHIORO

GÉRARD A. MAUGIN

ROBERTO NATALINI PATRIZIO NEFF

ANDREY PIATNITSKI

ERRICO PRESUTTI

MARIO PULVIRENTI

LUCIO RUSSO

Miguel A. F. SANJUAN

PATRICK SElvadurai

ALEXANDER P. SEYRANIAN

MIROSLAV ŠILHAVÝ

GUIDO SWEERS

ANTOINETTE TORDESILLAS

LEV TRUSKINOVSKY

JUAN J. L. VELÁZQUEZ VINCENZO VESPRI ANGELO VULPIANI msp.org/memocs

Università di Roma “La Sapienza”, Italia

Rutgers University, USA

(CO-CHAIR) Università di Roma "La Sapienza", Italia

(TREASURER) Università dell'Aquila, Italia

University of California at Davis, USA

(CO-CHAIR) Université Paris-Nord, France

Università dell'Aquila, Italy

École Polytechnique, France

DAMTP Cambridge, UK, and University of Vienna, Austria

(CHAIR MANAGING EDITOR) Univ. of Illinois at Urbana-Champaign, USA

Université du Sud Toulon-Var, France

University of California at Berkeley, USA

Universität Erlangen-Nürnberg, Germany

LMA CNRS Marseille, France

Università di Roma "La Sapienza", Italia

Università dell'Aquila, Italy

Université de Lyon-INSA (Institut National des Sciences Appliquées), France

(CHAIR MANAGING EDITOR) Univ. of Illinois at Urbana-Champaign, USA

Carnegie Mellon University, USA, and Bilkent University, Turkey

Otto-von-Guericke-Universität Magdeburg, Germany

Università di Roma "La Sapienza", Italia

University of Sheffield, UK

University of Novi Sad, Serbia

Wayne State University, USA

Université du Sud Toulon-Var, France

Università di Roma Tor Vergata, Italia

University of North Carolina at Chapel Hill, USA

Università di Pavia, Italia

Stanford University, USA

Institut Polytechnique de Grenoble, France

Università dell'Aquila, Italia

Università di Ferrara and International Research Center MEMOCS, Italia

Vanderbilt University, USA

Freie Universität Berlin, Germany

University of Texas at Austin, USA

Federation University and Australian National University, Australia

Université Aix-Marseille, France

Cornell University, USA

École des Mines, France

University of Western Ontario, Canada

Università dell' Aquila, Italy

Louisiana State University, USA

Università dell'Aquila, Italia

Université de Lyon-INSA (Institut National des Sciences Appliquées), France University of Pittsburgh, USA

Università di Roma "La Sapienza”, Italia

Université Paris VI, France

Istituto per le Applicazioni del Calcolo "M. Picone", Italy

Universität Duisburg-Essen, Germany

Narvik University College, Norway, Russia

Università di Roma Tor Vergata, Italy

Università di Roma "La Sapienza”, Italia

Università di Roma “Tor Vergata”, Italia

Universidad Rey Juan Carlos, Madrid, Spain

McGill University, Canada

Moscow State Lomonosov University, Russia

Academy of Sciences of the Czech Republic

Universität zu Köln, Germany

University of Melbourne, Australia

École Polytechnique, France

Bonn University, Germany

Università di Firenze, Italia

Università di Roma La Sapienza, Italia

MEMOCS (ISSN 2325-3444 electronic, 2326-7186 printed) is a journal of the International Research Center for the Mathematics and Mechanics of Complex Systems at the Università dell'Aquila, Italy.

Cover image: "Tangle” by $\odot$ John Horigan; produced using the Context Free program (contextfreeart.org).

PUBLISHED BY

7 mathematical sciences publishers

nonprofit scientific publishing

http://msp.org/

(C) 2017 Mathematical Sciences Publishers 
Mathematics and Mechanics of Complex Systems vol. 5 no. 3-4

Forms of the dissipation function for a class of viscoplastic 217 models

Massimo Cuomo

Tumor growth modelling by cellular automata

Ruben Interian, Reinaldo Rodríguez-Ramos, Fernando

Valdés-Ravelo, Ariel Ramírez-Torres, Celso C. Ribeiro and Aura Conci

A variational formulation for fuzzy analysis in continuum 261 mechanics

Rolf Mahnken

On the notion of stress in classical continuum mechanics

Simon R. Eugster and Christoph Glocker

MEMOCS is a journal of the International Research Center for the Mathematics and Mechanics of Complex Systems at the Università dell' Aquila, Italy.

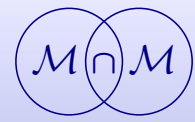

